\title{
Reorganization of a synthetic microbial consortium for one-step vitamin C fermentation
}

\author{
En-Xu Wang ${ }^{1,2}$, Ming-Zhu Ding ${ }^{1,2^{*}}$, Qian Ma ${ }^{1,2}$, Xiu-Tao Dong ${ }^{1,2}$ and Ying-Jin Yuan ${ }^{1,2}$
}

\begin{abstract}
Background: In the industry, the conventional two-step fermentation method was used to produce 2-keto-L-gulonic acid (2-KGA), the precursor of vitamin C, by three strains, namely, Gluconobacter oxydans, Bacillus spp. and Ketogulonicigenium vulgare. Despite its high production efficiency, the long incubation period and an additional second sterilization process inhibit the further development. Therefore, we aimed to reorganize a synthetic consortium of G. oxydans and $K$. vulgare for one-step fermentation of 2-KGA and enhance the symbiotic interaction between microorganisms to perform better.
\end{abstract}

Results: During the fermentation, competition for sorbose of G. oxydans arose when co-cultured with K. vulgare. In this study, the competition between the two microbes was alleviated and their mutualism was enhanced by deleting genes involved in sorbose metabolism of $G$. oxydans. In the engineered synthetic consortium $\left(\mathrm{H}_{6}+\mathrm{Kv}\right)$, the yield of 2-KGA (mol/mol) against D-sorbitol reached $89.7 \%$ within $36 \mathrm{~h}$, increased by $29.6 \%$. Furthermore, metabolomic analysis was used to verify the enhancement of the symbiotic relationship and to provide us potential strategies for improving the synthetic consortium. Additionally, a significant redistribution of metabolism occurred by co-culturing the $K$. vulgare with the engineered G. oxydans, mainly reflected in the increased TCA cycle, purine, and fatty acid metabolism.

Conclusions: We reorganized and optimized a synthetic consortium of G. oxydans and K. vulgare to produce 2-KGA directly from $\mathrm{D}$-sorbitol. The yield of 2-KGA was comparable to that of the conventional two-step fermentation. The metabolic interaction between the strains was further investigated by metabolomics, which verified the enhancement of the mutualism between the microbes and gave us a better understanding of the synthetic consortium.

Keywords: Synthetic microbial consortium, Reorganization, One-step fermentation, Interaction, Metabolomics

\section{Background}

As synthetic biology begins to address problems involved in the programing novel biological systems, engineering multicellular behavior is emerging as a key tool for building advanced synthetic systems that robustly perform complex behaviors [1]. In natural environments, microorganisms commonly exist as communities of multiple species that are capable of fulfilling more varied and complicate tasks than clonal populations [2]. Different members of a consortium assume different responsibilities,

\footnotetext{
*Correspondence: mzding@tju.edu.cn

2 SynBio Research Platform, Collaborative Innovation Center of Chemical Science and Engineering (Tianjin), Tianjin University, Tianjin 300072,

People's Republic of China

Full list of author information is available at the end of the article
}

increasing overall productivity and allowing for more complex behavior than that with a single cell or a monoculture. During the last decade, experimental efforts have been made to build and maintain the synthetic communities [3-5]. However, those studies were mostly concerned with the well-defined ideal models (e.g., mutualism, parasitism and commensalism, etc.). Whereas in industrially-relevant circumstances, the situation is more complex since the exchange of metabolites, energy, and informative signals with the environment should be taken into consideration, and the relationship among the strains is often too diverse to analyze. Therefore, increasing attention has been paid on the development of synthetic consortia in industry. Several microbial consortia were constructed for studying the cooperation of cells, 
enhancing the production of biofuel [6, 7], electricity [8] and even complex natural products [9], etc.

Generally, microorganisms interact with each other by exchanging biomolecules (e.g., proteins, nucleic acids and metabolites) and information signals via contact-based or contact-independent interaction [10]. Omics study can provide deep insights into the mechanism of metabolic crosstalk in a consortium at the global level and indicate the way to better understand the relationship between the species [11]. Researchers have adopted this approach to investigate the co-cultured microbial systems, including some previous studies about the metabolic cooperation of Bacillus megaterium and Ketogulonicigenium vulgare in two-step vitamin $C$ fermentation [12-16]. In this study, metabolomic analysis was used to better understand the specialization and cooperation between Gluconobacter oxydans and K. vulgare in the reorganized microbial consortium. These analyses verified the alleviation of competition and the enhancement of the symbiotic relationship, which provided us potential strategies for further construction of the microbes.

Chen et al. [17] declared the fundamental power of cell specialization and cooperation in the consortia. It is a powerful reminder that the communities are frequently more than the sum of their parts. In nature, microbes can form interacting communities to accomplish chemically difficult tasks through division of labor among different species [18]. In this study, the industrial vitamin $\mathrm{C}$ fermentation was taken as an example. The conventional two-step fermentation method was used to produce 2-keto-L-gulonic acid (2-KGA), the precursor of vitamin C, by three strains (G. oxydans, Bacillus spp. and $K$. vulgare). During the second step, $K$. vulgare is responsible for the biosynthesis of 2-KGA from L-sorbose, and Bacillus spp., as a companion, promotes the growth and production efficiency of $K$. vulgare. Despite its high production efficiency, the long incubation period and an additional second sterilization process of the two-step fermentation inhibit the further development of industrial production. Hence, we demonstrated the concept of reconstituting a heterologous metabolic pathway in a microbial partnership with G. oxydans and K. vulgare, where 2-KGA was produced directly from D-sorbitol. Furthermore, two genes involved in sorbose metabolism from G. oxydans were knocked out to alleviate the competition for sorbose of G. oxydans. The yield of 2-KGA $(\mathrm{mol} / \mathrm{mol})$ against D-sorbitol reached $89.7 \%(76.6 \mathrm{~g} / \mathrm{L})$ within $36 \mathrm{~h}$, which enabled an $29.6 \%$ increase compared to the original consortium $69.3 \%(59.1 \mathrm{~g} / \mathrm{L})$. Additionally, simplifying metabolic pathway may remove some negative effects for the microbes and increase the metabolic efficiency, which makes up for the mismatch of the consortium and enhances the cell-cell interaction. Hence, metabolomic analysis was used to provide a clear and comprehensive description of the physiological relationship between them, which was the key issue of this one-step fermentation. Compared with the conventional two-step fermentation process, this new route of one-step fermentation can potentially revolutionize the industrial-scale production of vitamin C.

\section{Methods}

\section{Strains}

The two industrial strains, G. oxydans and $K$. vulgare, used in this study were generously supplied by Welcome Pharmaceutical Co., Ltd. North China Pharmaceutical Group (Shijiazhuang, China). The stepwise construction of mutant $G$. oxydans strains with target gene deletion by homologous recombination is shown in Additional file 1: Figure S1. Escherchia coli DH5 $\alpha$ strain, purchased from Takara (Dalian, China), was used as an intermediate host for plasmid construction and propagation. Firstly, a promoter tufB and the recombination flanks of the target genes were individually amplified from the genome of $G$. oxydans. Then a selection marker gen or tet was amplified from two broad-host vectors. After that, all these four fragments were ligated through overlap extension PCR (OE PCR), forming gene deletion cassettes. During the whole process, all fragments of different cassettes were cloned into E. coli DH5 $\alpha$ using standard methods with a cloning vector $p E A S Y$-Blunt (TransGene Biotech Co., Ltd. Beijing). Sequentially, we transformed the cassettes into G. oxydans by electroporation and selected the correct clones from the agar plates with $210 \mu \mathrm{g} / \mathrm{ml}$ gentamycin or $25 \mu \mathrm{g} / \mathrm{ml}$ tetracycline. All the engineered bacterial strains got in this study were described in Table 1.

\section{Medium and culture conditions}

All E. coli strains were cultivated in Luria-Broth (LB) medium at $37{ }^{\circ} \mathrm{C}$. The $\mathrm{D}$-sorbitol/L-sorbose seed culture medium for the mono-culture of G. oxydans and

\begin{tabular}{|c|c|}
\hline Strains (abbreviations) & Target genes deleted \\
\hline SyBE_Go00010308 $\left(\mathrm{H}_{1}\right)$ & $\begin{array}{l}\text { B932_0664: FAD-dependent L-sorbose 1-dehy- } \\
\text { drogenase }\end{array}$ \\
\hline SyBE_Go00010309 $\left(\mathrm{H}_{2}\right)$ & $\begin{array}{l}\text { B932_1330: NADPH-dependent L-sorbose } \\
\text { reductase }\end{array}$ \\
\hline SyBE_Go00010310 $\left(\mathrm{H}_{3}\right)$ & B932_1370: PTS system transporter subunit IIA \\
\hline SyBE_Go00010311 $\left(\mathrm{H}_{4}\right)$ & $\begin{array}{l}\text { B932_1684: NADPH-dependent L-sorbose } \\
\text { reductase }\end{array}$ \\
\hline SyBE_Go00010312 $\left(\mathrm{H}_{5}\right)$ & $\begin{array}{l}\text { B932_3022: NADPH-dependent L-sorbose } \\
\text { reductase }\end{array}$ \\
\hline SyBE_Go00010313 $\left(\mathrm{H}_{6}\right)$ & B932_1330 and B932_1370 \\
\hline
\end{tabular}


K. vulgare was composed of $20 \mathrm{~g} / \mathrm{L}$ D-sorbitol (for $G$. oxydans) or L-sorbose (for $K$. vulgare), $10 \mathrm{~g} / \mathrm{L}$ peptone, $3 \mathrm{~g} / \mathrm{L}$ corn-steep liquor (CSL), $3 \mathrm{~g} / \mathrm{L}$ beef extract, $3 \mathrm{~g} / \mathrm{L}$ yeast extract, $1 \mathrm{~g} / \mathrm{L}$ urea, $1 \mathrm{~g} / \mathrm{L} \mathrm{KH}_{2} \mathrm{PO}_{4}, 1 \mathrm{~g} / \mathrm{L} \mathrm{CaCO}_{3}$ and $0.2 \mathrm{~g} / \mathrm{L} \mathrm{MgSO}_{4} \cdot 7 \mathrm{H}_{2} \mathrm{O}$. The fermentation medium for the one-step co-culture contained $80 \mathrm{~g} / \mathrm{L} \mathrm{D}$-sorbitol, $10 \mathrm{~g} / \mathrm{L}$ CSL, $12 \mathrm{~g} / \mathrm{L}$ urea, $1 \mathrm{~g} / \mathrm{L} \mathrm{KH}_{2} \mathrm{PO}_{4}, 1 \mathrm{~g} / \mathrm{L} \mathrm{CaCO}{ }_{3}$ and $0.2 \mathrm{~g} / \mathrm{L} \mathrm{MgSO}_{4} \cdot 7 \mathrm{H}_{2} \mathrm{O}$. The $\mathrm{pH}$ values of the medium were maintained at 7.0 by the addition of $\mathrm{NaOH}$.

The mono-culture of $G$. oxydans and $K$. vulgare, as seed for the subsequent co-culture fermentation, were cultivated in $250 \mathrm{ml}$ flasks with $50 \mathrm{ml} \mathrm{D-sorbitol/L-sorbose}$ seed cultures at $30{ }^{\circ} \mathrm{C}$ and $250 \mathrm{rpm}$ for $24 \mathrm{~h}$. The $\mathrm{OD}_{600}$ of $G$. oxydans and $K$. vulgare in the seed culture reached about 5.5 and 3.0, respectively. Whereafter, these two strains were simultaneously inoculated into a $5 \mathrm{~L}$ jar fermentor (Bailun Bio-technology Co. Ltd., Shanghai) with $3 \mathrm{~L}$ fermentation medium. The inoculation ratio $(\%, \mathrm{v} / \mathrm{v})$ of G. oxydans and $K$. vulgare, agitation speed and aeration rate were optimized. The initial inoculum ratio of $G$. oxydans and $K$. vulgare was 4:1. pH value and temperature of the fed-batch fermentation were automatically controlled at 7.0 and $30{ }^{\circ} \mathrm{C}$. And the agitation speed was controlled at $500 \mathrm{rpm}$ with the aeration rate of $1.5 \mathrm{vvm}$.

\section{Analysis of population of each species}

Co- and mono-cultured community samples were collected from fermentations at $0,4,8,14,21,28$ and $32 \mathrm{~h}$ after inoculation. The genomic DNA was extracted from the samples with the TIANamp Bacteria DNA Kit (Tiangen Biotech, China). RealMasterMix (SYBR Green) was used and the quantitative PCR reactions were performed on Light-Cycler 480 with the primers designed based on 16S rDNA of each species 5'-CGATGTGTGCTGGATGTTGGG-3' and 5'-TCTGA ACCGGTCCTCCCCATG-3' for G. oxydans, and 5'-A ATGCCAGTCGTCAGGTTGCTT-3' ${ }^{\prime}$ and $5^{\prime}$-CTAGGCC GGTCCTGTAATGTCA-3' for $K$. vulgare. The amount of genome of each species was computed by comparison with a standard curve from pure cultures analyzed with the same manner.

\section{Transcriptional analysis of relevant genes in co- and mono-cultured systems}

The transcriptional expression level of the genes in coand mono-cultured systems at different sampling times was evaluated by qPCR. All the data were normalized to $16 \mathrm{~S}$ rDNA of each species. The entire RNA was extracted from the samples with the ApexPrep RNA Miniprep Kit (APExBio). HiTaq EvaGreen qPCR MasterMix (APExBio) was used and the quantitative PCR reactions were performed on a CFX96 real time PCR system (Bio-Rad) with a total volume of $20 \mu \mathrm{L}$ containing diluted cDNA
$(2 \mu \mathrm{L})$, qPCR MasterMix $(10 \mu \mathrm{L})$, and forward primer and reverse primer $(0.8 \mu \mathrm{L})$.

\section{Sample preparation and metabolites analysis}

The samples from different co- and mono-cultured fermentations were collected at 4, 8, 14, 21 and $28 \mathrm{~h}$ after inoculation. These five time points primarily represented the lag phase, the early exponential phase, the middle exponential phase, the late exponential phase and the stationary phase of community. The concentrations of extracellular D-sorbitol, L-sorbose and 2-KGA were analyzed by HPLC (Waters Corp., USA) with a refractive index detector. $\mathrm{H}_{2} \mathrm{SO}_{4}(5 \mathrm{mM})$ was used as the mobile phase on an Aminex HPX-87H column (BioRad, CA) at the temperature of $65^{\circ} \mathrm{C}$ with a flow rate of $0.6 \mathrm{ml} / \mathrm{min}$.

The intracellular metabolites were extracted and derivatizated according to our previous procedure [19]. Gas chromatography time-of-flight mass spectrometry (GC-TOF/MS, Waters Corp., USA) was applied to detect the metabolites in different samples, as described by Ding et al. [20] with identical chromatographic conditions. One microlitre sample was injected with a split ratio of 1:1 into GC, equipped with a fused-silica capillary column (DB-5MS, $30 \mathrm{~m} \times 0.25 \mathrm{~mm}$ i.d., $0.25 \mu \mathrm{m}$, J\&W Scientific, Folsom, CA, USA). After a 2 min delay at $70{ }^{\circ} \mathrm{C}$, the oven temperature program increased to $290{ }^{\circ} \mathrm{C}$ at $5{ }^{\circ} \mathrm{C} / \mathrm{min}$, holding for $3 \mathrm{~min}$. The temperature of the transfer line and the ion source was 280 and $250{ }^{\circ} \mathrm{C}$, respectively. Helium (99.9995\%) was used as the carrier gas under a constant pressure of $91 \mathrm{kPa}$. The solvent delay was $5 \mathrm{~min}$. Ions were generated by a $70 \mathrm{eV}$ electron beam at an ionization current of $40 \mu \mathrm{A}$. Two spectra were recorded per second in the mass range of $50-800 \mathrm{~m} / \mathrm{z}$ with DRE function.

Principal component analysis (PCA) and pathway enrichment analysis were performed by MetaboAnalyst 3.0 (http://www.metaboanalyst.ca/MetaboAnalyst/faces/ home.xhtml) [21].

\section{Results and discussion}

Reorganization of a synthetic microbial consortium for one-step vitamin C fermentation

Because of the survival traits of $K$. vulgare, the companion bacterium is not confined to Bacillus spp., some other bacteria, such as Xanthomonas maltophilia [22], can also be a good partner. Considering these conditions, Bacillus spp. was removed in this study and a synthetic consortium of G. oxydans-K. vulgare producing 2-KGA directly from D-sorbitol was constructed (Additional file 1: Figure S2a). The relationship within this synthetic microbial consortium was analyzed for further study. The whole fermentation process of G. oxydans is divided into two stages on the basis of D-sorbitol assimilation. In the 
co-culture system, the relationship turns from the commensalism of the first stage to the competition of the second stage, in accordance with the D-sorbitol consumption by G. oxydans (Fig. 1a-c).

The titer of 2-KGA by this consortium was only $12.9 \mathrm{~g} / \mathrm{L}$ within $36 \mathrm{~h}$, and the yield was $15.0 \%$, which was much lower than that of the industrial two-step fermentation process (about $90 \%$ ). In order to improve the performance of this one-step fermentation process, many optimization attempts have been made, including modification of the inoculation ratio, agitation speed, and aeration rate. In this way, the titer of 2-KGA reached to $59.1 \mathrm{~g} / \mathrm{L}$ within $28 \mathrm{~h}$, which shorten the fermentation time by about $40 \%$ (Additional file 1: Figure $\mathrm{S} 2 \mathrm{~b})$. Whereas in the control experiment in which only K. vulgare (Fig. 1d) or G. oxydans (data not shown) was cultured, no 2-KGA was produced. These results showed that L-sorbose produced by $G$. oxydans diffused into $K$. vulgare cells and was subsequently oxidized. However, though the optimization of fermentation conditions indeed improved the titer and yield of 2-KGA, the natural limitation of L-sorbose consumption by G. oxydans in this consortium cannot be overcome without genetic modification. In this study, G. oxydans was cultivated in D-sorbitol seed culture medium and the composition of the culture broth from at different time points during the fermentation was measured by HPLC. We found that L-sorbose cannot be consumed until D-sorbitol was exhausted after $12 \mathrm{~h}$ in the mono-culture of G. oxydans, which matched the conclusion drawn by Soemphol et al. [23]. Then the accumulation of an unknown byproduct was detected while L-sorbose was consumed (Fig. 1e), which would reduce the 2-KGA production and make the efficiency too low to fully meet industrial requirements. In industrial fermentation, even one percent loss of carbon source will cause a significant financial burden. Therefore, we further optimized this two-strain consortium by alleviating the metabolic competition of G. oxydans with $K$. vulgare for sorbose, which was helpful for establishing a better homeostasis between microbes and making them work better together.

\section{The relationship optimization of $G$. oxydans-K. vulgare consortium}

The core process for the synthetic microbial consortium we designed in this study was the conversion of L-sorbose, the substrate for $K$. vulgare to synthesize the final product 2-KGA. After screening the genome information of G. oxydans [24], five relevant genes (Table 1) encoding FAD-dependent L-sorbose 1-dehydrogenase, NADPHdependent L-sorbose reductase, and PTS system transporter subunit IIA in the L-sorbose consumption pathway were deleted, respectively. And five engineered
G. oxydans strains, namely $\mathrm{H}_{1}, \mathrm{H}_{2}, \mathrm{H}_{3}, \mathrm{H}_{4}$, and $\mathrm{H}_{5}$ (Fig. 2a), were obtained with the method mentioned in "Strains" section. Fermentations of each engineered strains were carried out in flasks and jar fermentors to test the effect of the gene deletions. Compared with $G$. oxydans, the engineered $\mathrm{H}_{2}, \mathrm{H}_{3}, \mathrm{H}_{4}$, and $\mathrm{H}_{5}$ significantly slowed down the consumption of $\mathrm{L}$-sorbose and increased the level of L-sorbose left in the broth after $30 \mathrm{~h}$ cultivation in L-sorbose seed culture medium in flasks (Fig. 2b). Among them, $\mathrm{H}_{2}$ and $\mathrm{H}_{3}$ were considered the most effective candidates for reducing sorbose utilization in $G$. oxydans. Consequently, a double mutant strain $\left(\mathrm{H}_{6}\right)$ was constructed by further deletion of NADPH-dependent L-sorbose reductase (B932_1330) in $\mathrm{H}_{3}$ to perform better for 2-KGA production in the consortium. After that, $\mathrm{H}_{2}$, $\mathrm{H}_{3}$ and $\mathrm{H}_{6}$ were individually co-cultured with $K$. vulgare, forming consortia $\mathrm{H}_{2}+\mathrm{Kv}, \mathrm{H}_{3}+\mathrm{Kv}$ and $\mathrm{H}_{6}+\mathrm{Kv}$, in the medium contained $8 \% \mathrm{D}$-sorbitol as substrate. The alleviation of competition and the enhancement of mutualism were verified by the undetected byproduct (Fig. 2c), the level of remaining sorbose in the broth (Fig. 2d) and the titer of 2-KGA (Fig. 2e). As a result, $\mathrm{H}_{2}, \mathrm{H}_{3}$ and $\mathrm{H}_{6}$ enabled an 18.6, 15.2 and $29.6 \%$ increase in the production of 2-KGA (70.1, 68.1 and $76.6 \mathrm{~g} / \mathrm{L})$ respectively compared to the primary consortium Go $+\mathrm{Kv}(59.1 \mathrm{~g} / \mathrm{L})$ after 28 or $36 \mathrm{~h}$ of cultivation. The relevant data of this study was compared with that of the conventional two-step fermentation (Additional file 1: Table S1). On one hand, the yield of 2-KGA was about 99 and $91 \%$ for each stage with $8 \%$ D-sorbitol as substrate in the two-step fermentation process. While in our study, it reached $89.7 \%$ with the same amount substrate and shortened the fermentation time by about $25 \%$. On the other hand, our route eliminated the need for a second sterilization process, where the rate of equipment utilization can be significantly improved and the production cost can be notably saved.

The optimization of the relationship between the two microbes, G. oxydans and K. vulgare, was further studied. The consortia population compositions throughout the process were analyzed to validate the variation of the relationship. Figure $2 \mathrm{f}$ and $\mathrm{g}$ showed the relative density of different microbes in co- and mono-cultured systems. We found that the $K$. vulgare in the engineered consortium $\mathrm{H}_{2}+\mathrm{Kv}$ also showed a better growth than that in the primary consortium Go $+\mathrm{Kv}$ (Fig. 2f), coupled with the higher production of 2-KGA in $\mathrm{H}_{2}+\mathrm{Kv}$. Meanwhile, the growth levels of engineered $\mathrm{H}_{2}$ and $G$. oxydans were similar in mono-culture. While after the introduction of $K$. vulgare, the engineered $\mathrm{H}_{2}$ grew much faster than the wild type since $8 \mathrm{~h}$ after inoculation and reached almost twice of the wild type after $28 \mathrm{~h}$ (Fig. $2 \mathrm{~g}$ ). In the present study, another interesting phenomenon about the initial inoculum ratio of G. oxydans to $K$. vulgare has 
a
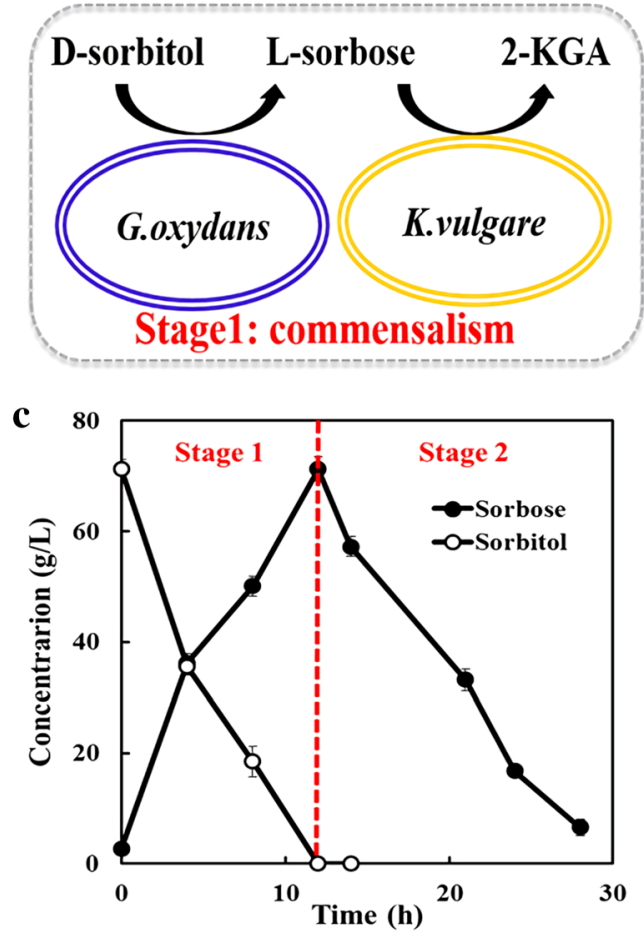

b

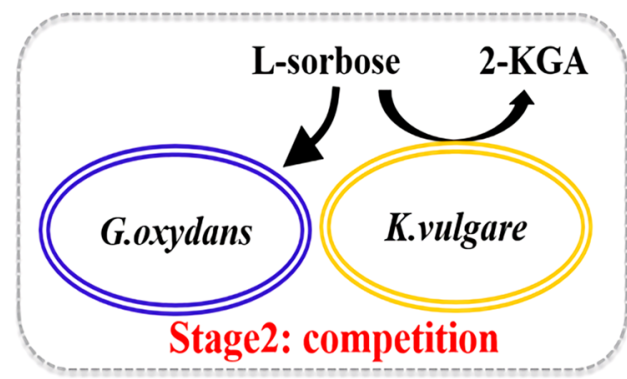

d

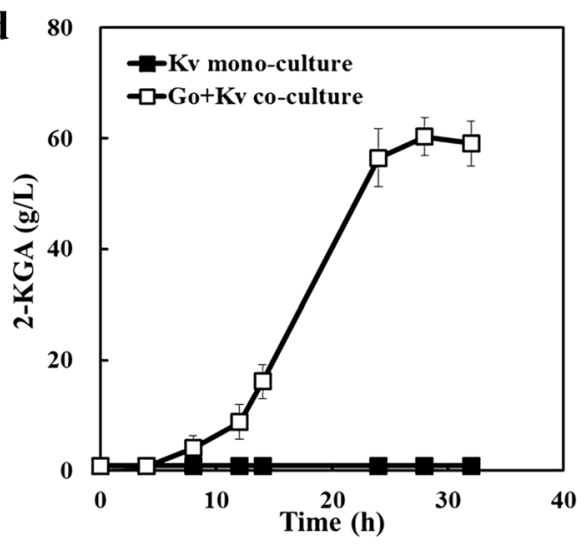

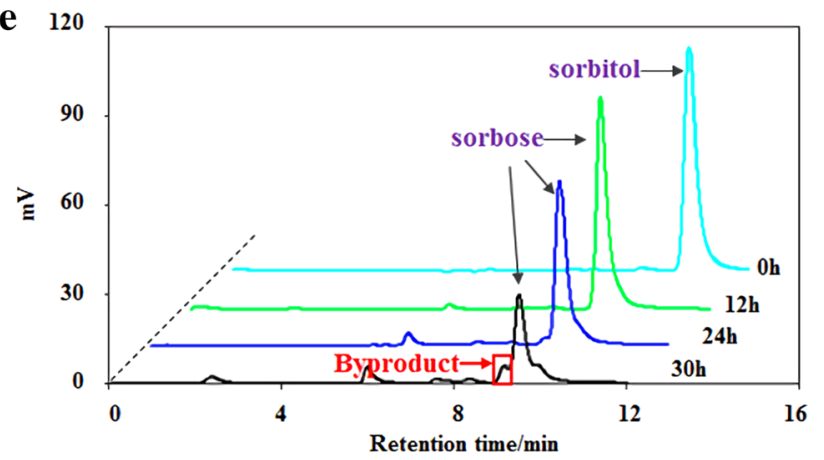

Fig. 1 The relationship analysis of G. oxydans-K. vulgare consortium. a A commensalistic G. oxydans-K. vulgare consortium for production of 2-KGA within stage $1 ; \mathbf{b}$ a competitive $G$. oxydans-K. vulgare consortium for production of 2-KGA within stage 2; c the division of two stages in accordance with the D-sorbitol consumption by G. oxydans. d only the co-culture produces 2-KGA; e chromatograms from HPLC analysis of D-sorbitol assimilation catalyzed by G. oxydans, the peak in the red box in shows the unknown byproduct produced by G. oxydans accompany with the sorbose assimilation

been found. The inoculum ratio (\%, v/v) of G. oxydans to $K$. vulgare was firstly set as 1:4 because of the growth defect of $K$. vulgare. Due to a low yield of 2-KGA, we then adjusted it to $4: 1$, which led to a great improvement in 2-KGA productivity (data not shown). This appears to be a counterintuitive finding that high ratio of inoculated G. oxydans was beneficial for the synthetic consortium. We speculated that because of the survival traits of $K$. vulgare, more G. oxydans were needed to provide more nutrients for the growth and productivity of $K$. vulgare. It was found that G. oxydans was the most populous consortium member throughout the whole process. However the ratio of G. oxydans to K. vulgare decreased during the fermentation in the engineered consortium, which was contrary to the original consortium Go $+\mathrm{Kv}$. From this point of view, the engineered $\mathrm{H}_{2}$ promoted the growth and productivity of $K$. vulgare and the latter stimulated the growth of $\mathrm{H}_{2}$ in return. We hypothesized that there was more interaction of biomolecules or information signals between the two microbes in this mutualistic G. oxydans-K. vulgare consortium (Fig. 2h) compared with the primary competitive consortium. 
$\boldsymbol{a}$

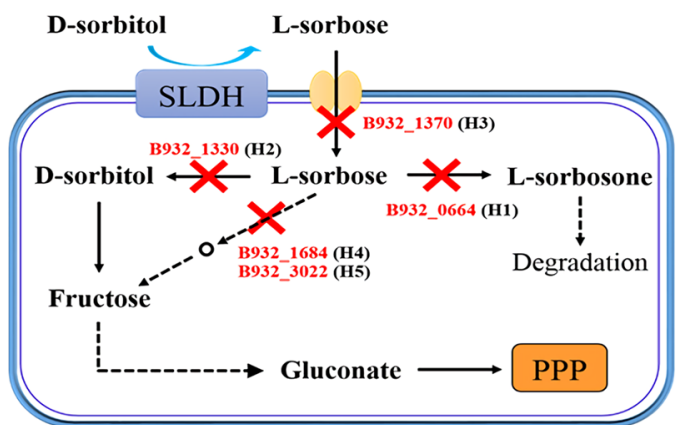

c

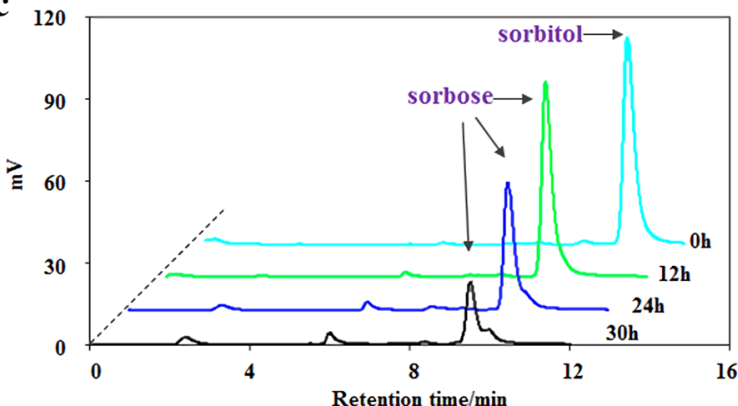

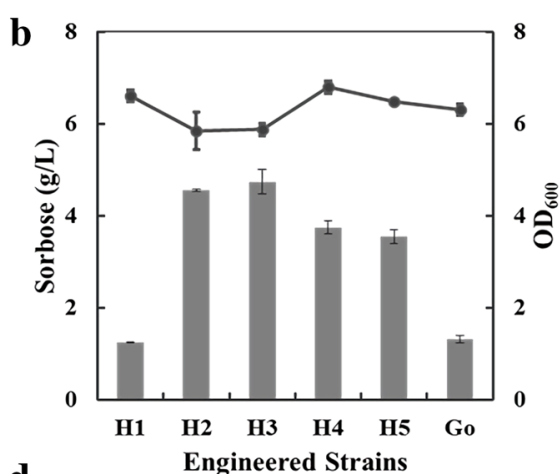

d 80

Engineered Strains

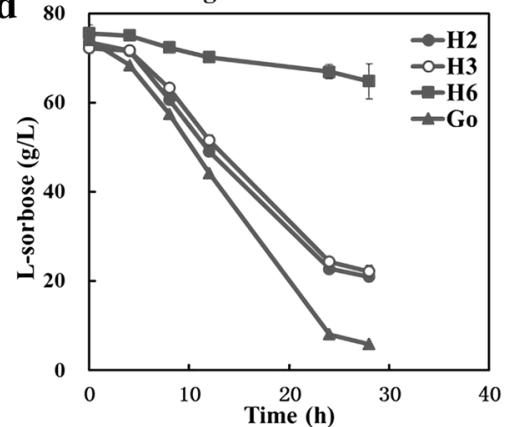

f

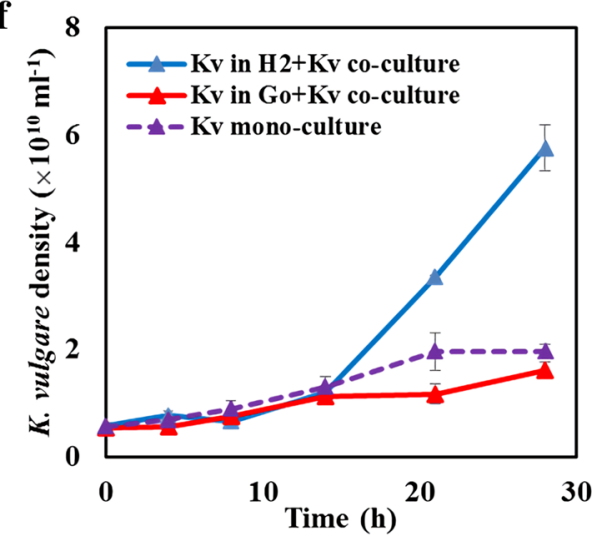

h

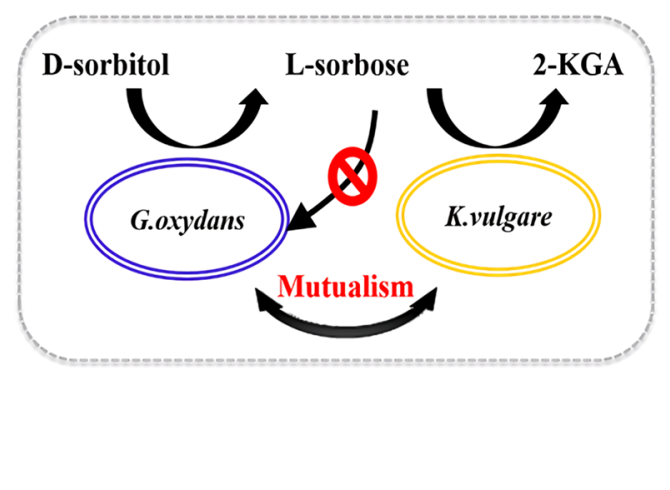

Fig. 2 The relationship analysis of the reorganized G. oxydans-K. vulgare consortium. a Sorbose metabolism in G. oxydans, red words indicate the genes involved in sorbose metabolism in G. oxydans; $\mathbf{b}$ effect of engineered strains on L-sorbose assimilation in the flasks; c chromatograms from HPLC analysis of D-sorbitol assimilation catalyzed by engineered $\mathrm{H}_{2}$, where no byproduct can be detected with the sorbose assimilation; $\mathbf{d}$ effect of engineered strain on L-sorbose assimilation in the jar fermentors; e effect of engineered $\mathrm{G}$. oxydans strains on 2-KGA accumulation in the synthetic consortium; $\mathbf{f}$ K. vulgare density in co- and mono-culture; $\mathbf{g}$ G. oxydans and engineered $\mathrm{H}_{2}$ density in co- and mono-culture; $\mathbf{h}$ a mutualistic $\mathrm{G}$. oxydans-K. vulgare consortium for production of 2-KGA 
Hence, metabolomic analysis of the different consortium should be done for a comprehensive description of the relationship optimization between the members.

\section{Metabolomic analysis on the relationship optimization of $G$. oxydans-K. vulgare consortium}

Simplifying the sorbose metabolic pathway will affect not only itself alone, but also other related metabolic characteristics. Thus, the metabolome of the engineered consortium $\mathrm{H}_{2}+\mathrm{Kv}$ was compared with the primary consortium $\mathrm{Go}+\mathrm{Kv}$ to better understand the metabolic changes. It was found by PCA that the metabolomic data of the consortia Go $+\mathrm{Kv}$ and $\mathrm{H}_{2}+\mathrm{Kv}$ at different sampling times $(4,8,14,21,28 \mathrm{~h})$ grouped clearly, respectively. An interesting phenomenon was that the metabolism of consortia Go $+\mathrm{Kv}$ and $\mathrm{H}_{2}+\mathrm{Kv}$ had opposite trajectories over time (Fig. 3a, b). It indicated that the metabolic characteristics of the consortium changed after the G. oxydans was replaced by the engineered $\mathrm{H}_{2}$. Pathway enrichment analysis was then carried out on the metabolomic data of consortia Go $+\mathrm{Kv}$ and $\mathrm{H}_{2}+\mathrm{Kv}$ (Fig. 3c, d), we found that the glycine, serine and threonine metabolism pathways and the pyruvate metabolism pathway were among the most significantly impacted in both $\mathrm{Go}+\mathrm{Kv}$ and $\mathrm{H}_{2}+\mathrm{Kv}$ consortia. Besides, glycerophospholipid and glycerolipid metabolism were also demonstrated significant change in the $\mathrm{H}_{2}+\mathrm{Kv}$ consortium compared to Go $+\mathrm{Kv}$. Furthermore, the metabolism significantly impacted in two consortia with different metabolic trajectories indicated that the relationship between the two strains in both consortia were different.

\section{Improved amino acids metabolism in G. oxydans-K. vulgare consortium}

A complete understanding of microbial metabolism should extend from the properties of individual strain in pure culture to the combinatorial interactions supported by complex communities. Metabolic levels of G. oxydans in monoculture were compared with those in the consortium of G. oxydans and K. vulgare to better understand the metabolic effects by $K$. vulgare, and the metabolic interaction between $G$. oxydans and $K$. vulgare in the synthetic microbial consortium. Our metabolomics analysis showed that the metabolism of the TCA cycle, amino acids, purines and free fatty acids were all significantly affected by the introduction of $K$. vulgare to the fermentation of sorbitol by G. oxydans or $\mathrm{H}_{2}$. The variations of these metabolites of the consortium were compared with those of Go or $\mathrm{H}_{2}$, and the fold changes of metabolites in the consortium after engineering relative to those in primary one are shown in Fig. 3e. In this study, when part of $G$. oxydans was replaced by $K$. vulgare for fermentation, the levels of most intracellular amino acids were found to change a lot. It was reported by Liu et al. [25] that the genes contributing to the de novo biosynthesis of histidine (His), glycine (Gly), lysine (Lys), proline (Pro), threonine (Thr), methionine (Met), leucine (Leu), and isoleucine (Ile) were absent in $K$. vulgare. Thus, it was supposed that the amino acids levels in the consortium would be lower than that in G. oxydans monoculture. However, five of the eight deficient amino acids including His, Pro, Thr, Leu and Ile in the consortium represented higher levels in synthetic consortium, suggesting that G. oxydans synthetized more of these amino acids, allowing for the better growth and production of $K$. vulgare. As a consequence, we speculated that the proper supplement of these amino acids would promote better growth and production of $K . v u l-$ gare, which improved the interaction of two strains during fermentation. In order to prove this hypothesis, we investigated the effect of these amino acids on the productivity of the consortium. These five amino acids were added into the fermentation medium individually and they did enhance the ability of 2-KGA productivity to some extent as expected (Fig. 4a). Next, a mixture of His, Pro, Thr, Leu and Ile, with a final concentration of 0.7, 0.3, 0.5, 0.1, $0.5 \mathrm{~g} / \mathrm{L}$, respectively, was added to the consortium of $G$. oxydans and $K$. vulgare. With the addition of these amino acids, the yield of 2-KGA in flask cultures after $36 \mathrm{~h}$ of cultivation reached $88.3 \%$, enjoying a $41.8 \%$ increase compared to the original consortium (62.3\%) with no addition of amino acids (Fig. 4a). In addition, the transcriptional expression level of histidinol-phosphatase, 1-pyrroline5-carboxylate reductase, homoserine kinase and 3-isopropylmalate dehydratase in co- and mono-cultured systems at different sampling time points was evaluated by qPCR for further evidence. It was found that 1-pyrroline-5-carboxylate reductase, homoserine kinase and 3-isopropylmalate dehydratase in synthetic consortium enjoyed a similar tendency (Fig. 4b-d). The transcriptional expression of these genes in $\mathrm{H}_{2}+\mathrm{Kv}$ consortium showed a highest level at the early point of exponential phase $(14 \mathrm{~h})$ as expected, which was in accordance with the tendency of growth and productivity of $K$. vulgare. However, the levels of these genes were much lower in Go $+\mathrm{Kv}$ consortium and no expression could be detected in the mono-culture of $K$. vulgare. From this point of view, it proved that with the stimulation of $K$. vulgare, $G$. oxydans synthetized more of these amino acids for better growth and production of $K$. vulgare.

\section{Improved purines metabolism in G. oxydans-K. vulgare consortium}

In addition to amino acid biosynthesis deficiencies, $K$. vulgare was reported to be insufficient in purine nucleotide biosynthesis $[26,27]$. Our previous study also found 

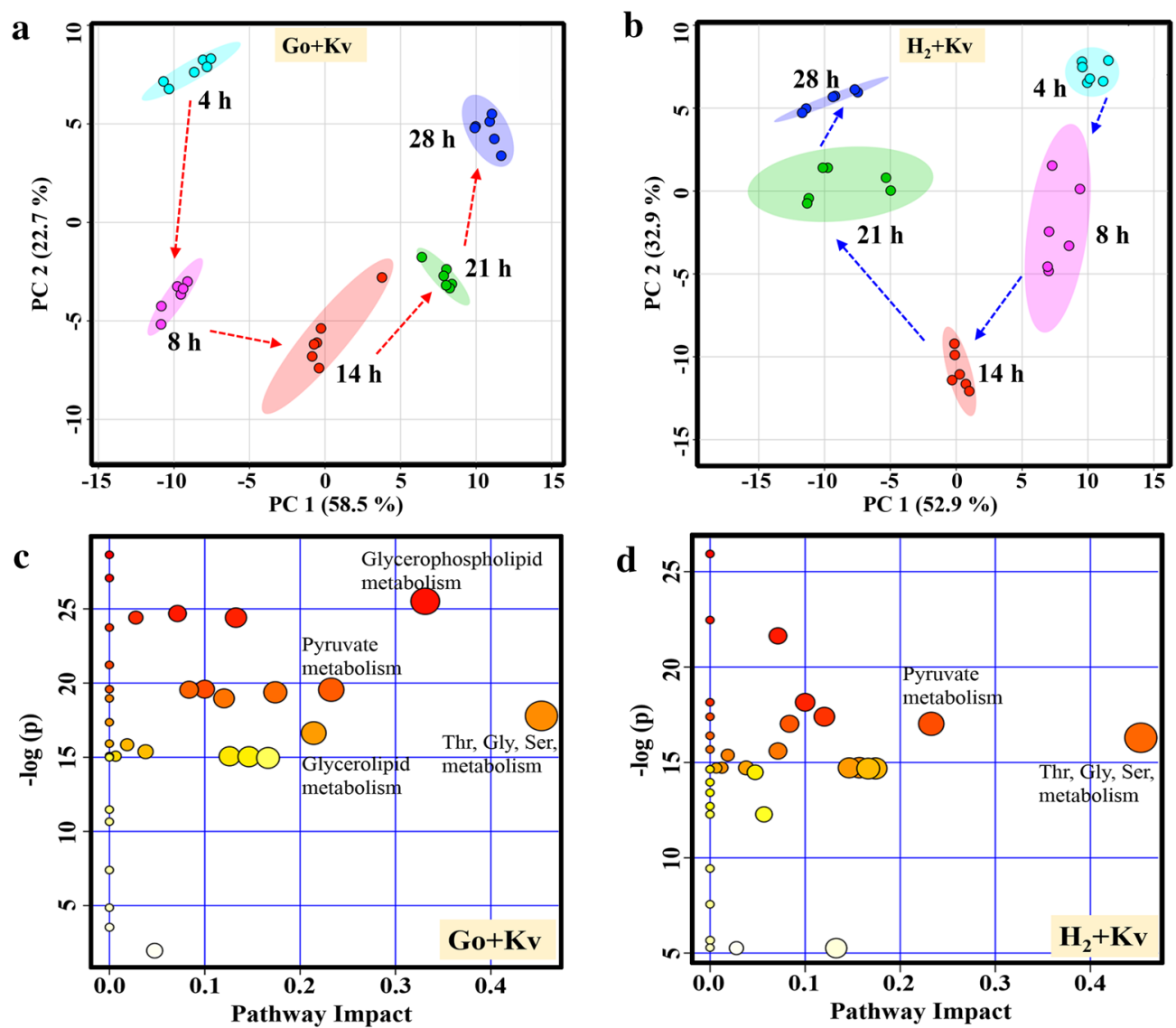

e

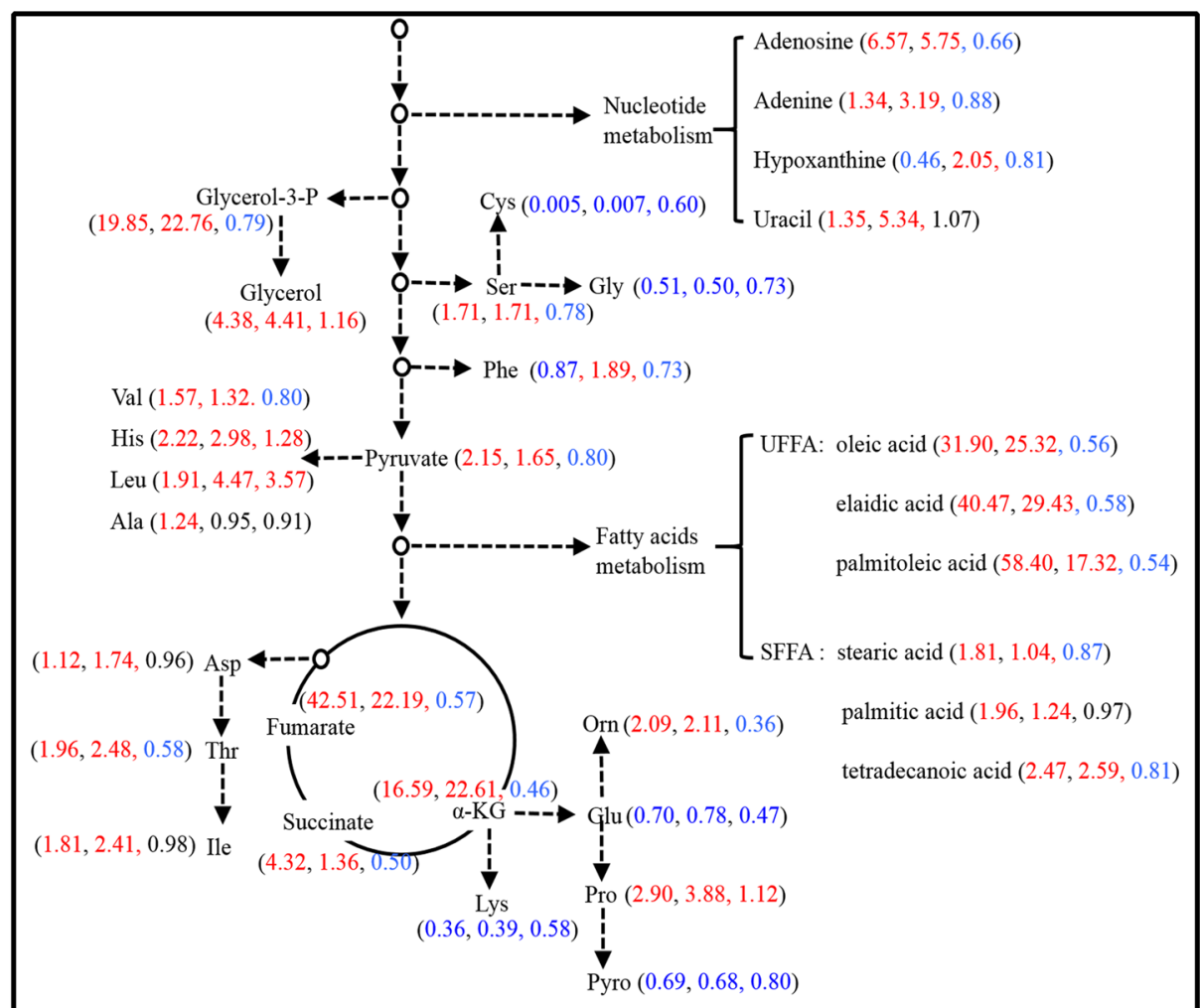


(See figure on previous page.)

Fig. 3 Metabolomic analysis on the relationship optimization of G. oxydans-K. vulgare consortium. a PCA score plot of time-series metabolomics data of Go + Kv; $\mathbf{b}$ PCA score plot of time-series metabolomics data of $\mathrm{H}_{2}+\mathrm{Kv}$; $\mathbf{c}$ Pathway enrichment analysis of consortium Go + Kv, circles represent all the matched pathways according to KEGG. The color and size of each circle is based on $P$ values ( $y$-axis) and pathway impact values ( $x$-axis), respectively. The darker the circle color, the more significant and coordinated changes of metabolites in the matched metabolic pathway. The bigger the circle size, the higher the centrality of its involved metabolites. $\mathbf{d}$ Pathway enrichment analysis of consortium $\mathrm{H}_{2}+\mathrm{Kv}_{\text {; }}$ e Fold changes of metabolites in central carbon metabolism and related metabolism. The numbers in the bracket from left to right indicated the fold changes of metabolite abundance in consortium $\mathrm{Go}+\mathrm{Kv}$ relative to $\mathrm{Go}$, in $\mathrm{H}_{2}+\mathrm{Kv}$ relative to $\mathrm{H}_{2}$, and in $\mathrm{H}_{2}+\mathrm{Kv}$ relative to $\mathrm{Go}+\mathrm{Kv}$, respectively. Red numbers indicate the increased levels, and blue numbers indicate the decreased levels

that supplement of purines did have certain positive effects on the cell growth and the 2-KGA productivity of $K$. vulgare. [12]. In this study, adenine and adenosine contents were both higher in consortia $\mathrm{Go}+\mathrm{Kv}$ and $\mathrm{H}_{2}+\mathrm{Kv}$ when compared with mono-cultured G. oxydans and $\mathrm{H}_{2}$, respectively (Fig. 3e). We also found that adenine and adenosine were undetected in mono-cultured $K$. vulgare. This suggests that G. oxydans provides these purines to $K$. vulgare when co-cultured, while in return, $K$. vulgare stimulates the biosynthesis of purines in G. oxydans. In addition, the levels of these purines decreased after engineering, which indicated the gene deletion did affect the biosynthesis of purines. However, the increase of the purines levels in co-cultured $\mathrm{H}_{2}+\mathrm{Kv}$ compared to the mono-cultured $\mathrm{H}_{2}$ was larger than that in co-cultured Go + Kv comparing to G. oxydans, which suggested that co-culturing with $K$. vulgare promoted the biosynthesis of purines more in the engineered G. oxydans than that
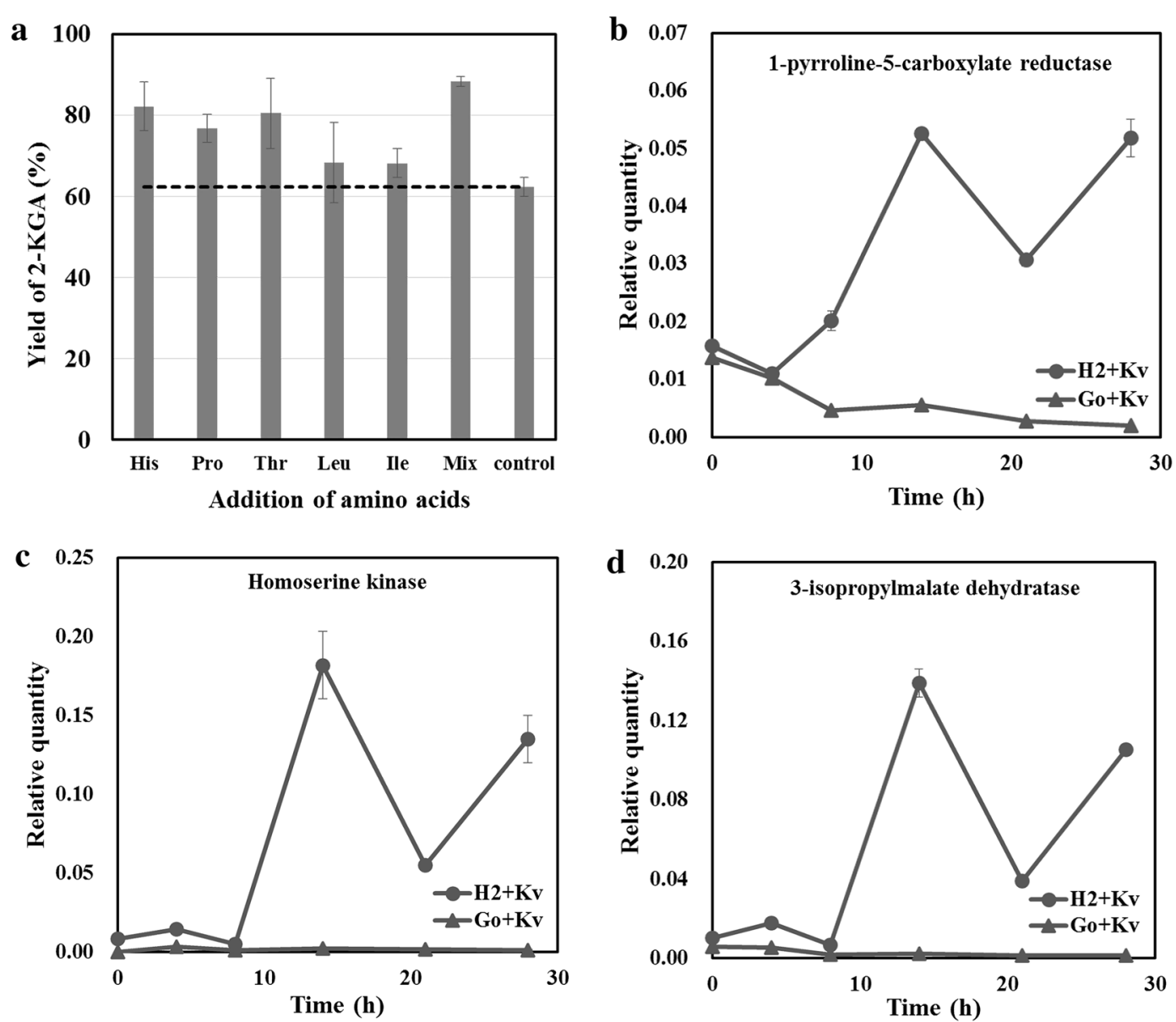

Fig. 4 Analysis of the improved amino acids metabolism in G. oxydans-K. vulgare consortium. a Effect of adding amino acids on the synthetic consortium in the flask; Transcriptional expression level of b 1-pyrroline-5-carboxylate reductase (EC 1.5.1.2); c Homoserine kinase (EC 2.7.1.39); d 3-isopropylmalate dehydratase (EC 4.2.1.33) in co-cultured systems. All the data were normalized to $16 \mathrm{~S}$ rDNA of each species 
in the wild type. Therefore, on one hand, we suggested that $G$. oxydans provided substrates and nutrients for $K$. vulgare. On the other hand, $K$. vulgare gave some feedback to stimulate the synthesis of nutrients in G. oxydans (Fig. 5).

\section{Improved fatty acids metabolism in G. oxydans-K. vulgare consortium}

In this study, all the detected free fatty acids represented higher levels in consortium samples than that in monocultured G. oxydans samples, especially the unsaturated fatty acids including oleic acid (18:1), elaidic acid (18:1), and palmitelaidic acid (16:1). All of these three fatty acids were presented at over 20-fold higher levels in both consortia compared to monoculture. Additionally, their levels in consortium Go $+\mathrm{Kv}$ were higher than those in $\mathrm{H}_{2}+\mathrm{Kv}$, respectively (Fig. 3e). It was reported that the increased unsaturated fatty acid level facilitated the stress defense [28]. Thus, we speculated that G. oxydans might be subjected to several stresses after co-cultured with $K$. vulgare, such as the changed growth environment caused by the metabolites secreted by $K$. vulgare. More unsaturated fatty acids were synthesized by $G$. oxydans to respond to the pressure of the co-culture conditions. Compared to Go $+\mathrm{Kv}$, the lower levels of unsaturated fatty acids in consortium $\mathrm{H}_{2}+\mathrm{Kv}$ suggested that the engineered $\mathrm{H}_{2}$ possessed preferable adaptability to the environment co-culture with $K$. vulgare. On the other hand, more unsaturated fatty acids would increase the cell membrane fluidity and permeability under unfavorable conditions by affecting the plasma membrane integrity, fluidity and function [29]. The dramatic increase in the levels of these unsaturated fatty acids may indicate that cells in this consortium increased their membrane permeability for exchanging more nutrients, which would promote the interaction between two strains.

\section{Conclusions}

In this study, a synthetic consortium for one-step vitamin $C$ fermentation was reorganized with $G$. oxydans and $K$. vulgare. Further optimization was carried out to alleviate the competition for sorbose of $G$. oxydans with $K$. vulgare. The yield of 2-KGA of this consortium reached $89.7 \%$ within $36 \mathrm{~h}$, which is comparable to the conventional two-step fermentation. The metabolic interaction between the strains was further investigated by metabolomics, which verified the enhancement of the mutualism between the microbes and gave us a better understanding of the synthetic consortium.

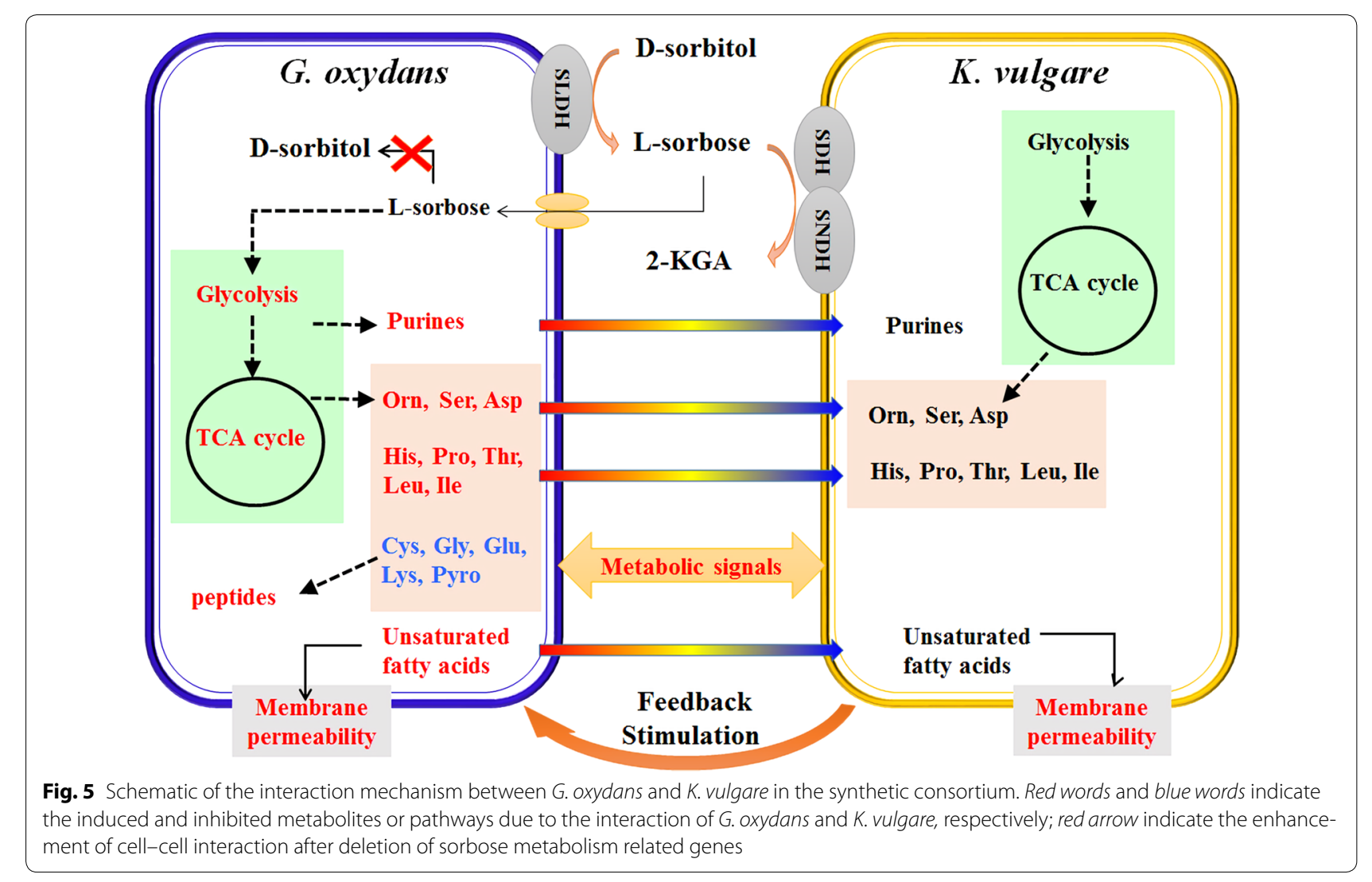




\section{Additional file}

Additional file 1: Table S1. Comparison of one-step and two-step fermentation process. Figure S1. Double-crossover homologous recombination schematic diagram. Figure S2. A new one-step fermentation route for production of 2-KGA. a Redesign of the conventional industrial fermentation route for one-step 2-KGA production; b 2-KGA accumulation in the synthetic consortium of G. oxydans-K. vulgare. Circle indicates the inoculation ratio of G. oxydans and K. vulgare was 1:4, the agitation speed was $400 \mathrm{rpm}$, the aeration rate was $1.0 \mathrm{vvm}$; triangle indicates the inoculation ratio of $G$. oxydans and $K$. vulgare was 1:2, the agitation speed was 400 $\mathrm{rpm}$, the aeration rate was $1.0 \mathrm{vvm}$; square indicates the inoculation ratio of $G$. oxydans and K. vulgare was 4:1, the agitation speed was $500 \mathrm{rpm}$, the aeration rate was $1.5 \mathrm{vvm}$

\section{Authors' contributions}

EXW, MZD and YJY designed the study and drafted the manuscript. EXW and MZD carried out the molecular genetic studies and metabolomics analysis. QM and XTD assisted in the fermentation and metabolomics analysis. YJY supervised the whole research and revised the manuscript. All authors read and approved the final manuscript.

\section{Author details}

${ }^{1}$ Key Laboratory of Systems Bioengineering (Ministry of Education), School of Chemical Engineering and Technology, Tianjin University, Tianjin 300072, People's Republic of China. ${ }^{2}$ SynBio Research Platform, Collaborative Innovation Center of Chemical Science and Engineering (Tianjin), Tianjin University, Tianjin 300072, People's Republic of China.

\section{Acknowledgements}

There is no conflict of interest among the authors. This work was funded by the Ministry of Science and Technology of China ("973" Program: 2014CB745100, "863" Program: 2012AA02A701), and the National Natural Science Foundation of China (Major Program: 21390203).

\section{Competing interests}

The authors declares that they have no competing interests.

Received: 19 November 2015 Accepted: 11 January 2016

Published online: 25 January 2016

\section{References}

1. Teague BP, Weiss R. Synthetic communities the sum of parts. Science. 2015;349:924-5.

2. Brune KD, Bayer TS. Engineering microbial consortia to enhance biomining and bioremediation. Front Microbiol. 2012;3:203.

3. Shou W, Ram S, Vilar JMG. Synthetic cooperation in engineered yeast populations. Proc Natl Acad Sci USA. 2007;104:1877-82.

4. Chuang JS, Rivoire O, Leibler S. Simpson's paradox in a synthetic microbial system. Science. 2009;323:272-5.

5. Waite AJ, Shou W. Adaptation to a new environment allows cooperators to purge cheaters stochastically. Proc Natl Acad Sci USA. 2012;109:19079-86.

6. Tsai SL, Goyal G, Chen W. Surface display of a functional minicellulosome by intracellular complementation using a synthetic yeast consortium and its application to cellulose hydrolysis and ethanol production. Appl Microbiol Biotechnol. 2010;76(22):7514-20.

7. Minty JJ, Singer ME, Scholz SA, Bae CH, Ahn JH, Foster CE, et al. Design and characterization of synthetic fungal-bacterial consortia for direct production of isobutanol from cellulosic biomass. Proc Natl Acad Sci USA. 2013;110:14592-7.
8. Rosenbaum MA, Bar HY, Beg QK, Segrèc D, Booth J, Cotta MA, Angenent LT. Shewanella oneidensis in a lactate-fed pure-culture and a glucose-fed co-culture with Lactococcus lactis with an electrode as electron acceptor. Bioresour Technol. 2011;102:2623-8.

9. Zhou K, Qiao K, Edgar S, Stephanopoulos G. Distributing a metabolic pathway among a microbial consortium enhances production of natural products. Nat Biotechnol. 2015. doi:10.1038/nbt.3095.

10. Phelan WV, Liu WT, Pogliano K, Dorrestein PC. Microbial metabolic exchange-the chemotype-to-phenotype link. Nat Chem Biol. 2012:8:26-35.

11. Faust K, Raes J. Microbial interactions: from networks to models. Nat Rev Microbiol. 2012;10:538-50.

12. Ma Q, Zhou J, Zhang WW, Meng XX, Sun JW, Yuan YJ. Integrated proteomic and metabolomic analysis of an artificial microbial community for two-step production of vitamin C. PLoS One. 2011;6:e26108.

13. Du J, Zhou J, Xue J, Song H, Yuan YJ. Metabolomic profiling elucidates community dynamics of the Ketogulonigenium vulgare-Bacillus megaterium consortium. Metabolomics. 2012:8:960-73.

14. Zhou J, Yi H, Wang LL, Zhang WW, Yuan YJ. Metabolomic analysis of the positive effects on Ketogulonigenium vulgare growth and 2-keto-L-gulonic acid production by reduced glutathione. OMICS. 2012;16:387-96.

15. Ding MZ, Zou Y, Song H, Yuan YJ. Metabolomic analysis of cooperative adaptation between co-cultured Bacillus cereus and Ketogulonigenium vulgare. PLoS One. 2014;9:e94889.

16. Song H, Ding MZ, Jia XQ, Ma Q, Yuan YJ. Synthetic microbial consortia: from systematic analysis to construction and applications. Chem Soc Rev. 2014;43:6954-81.

17. Chen Y, Kim JK, Hirning AJ, Josić K, Bennett MR. Emergent genetic oscillations in a synthetic microbial consortium. Science. 2015;349:986-9.

18. Agapakis CM, Boyle PM, Silver PA. Natural strategies for the spatial optimizationof metabolism in synthetic biology. Nat Chem Biol. 2012;8:527-35.

19. Ding MZ, Zhou X, Yuan YJ. Metabolome profiling reveals adaptive evolution of Saccharomyces cerevisiae during repeated vacuum fermentations. Metabolomics. 2010;6:42-55.

20. Ding MZ, Wang $X$, Yang $Y$, Yuan YJ. Comparative metabolic profiling of parental and inhibitors-tolerant yeasts during lignocellulosic ethanol fermentation. Metabolomics. 2012;8:232-43.

21. Xia J, Wishart DS. Web-based inference of biological patterns functions and pathways from metabolomic data using MetaboAnalyst. Nat Protoc 2011:6:743-60.

22. Takagi Y, Sugisawa T, Hoshino T. Continuous 2-Keto-L-gulonic acid fermentation by mixed culture of Ketogulonigenium vulgare DSM 4025 and Bacillus megaterium or Xanthomonas maltophilia. Appl Microbil Biotechnol. 2010:86:469-80.

23. Soemphol W, Toyama H, Moonmangmee D, Adachi O, Matsushita K. L-Sorbose reductase and its transcriptional regulator involved in L-sorbose utilization of Gluconobacter frateurii. J Bacteriol. 2007;189:4800-8.

24. Ge X, Zhao Y, Hou W, Zhang WC, Chen WW, Wang JH, et al. Complete Genome Sequence of the Industrial Strain Gluconobacter oxydans H24. Genome Announc. 2013;1:e00003-13.

25. Liu L, Chen K, Zhang J, Liu J, Chen J. Gelatin enhances 2-keto-I-gulonic acid production based on Ketogulonigenium vulgare genome annotation. J Biotechnol. 2011;156:182-7.

26. Leduc S, Troostembergh JC, Lebeault JM. Folate requirements of the 2-keto-L-gulonic acid-producing strain Ketogulonigenium vulgare LMP P-20356 in L-sorbose/CSL medium. Appl Microbiol Biotechnol. 2004:65:163-7.

27. Cai L, Yuan MQ, Li ZJ, Chen JC, Chen GQ. Genetic engineering of Ketogulonigenium vulgare for enhanced production of 2-keto-L-gulonic acid. J Biotechnol. 2012;157:320-5.

28. You KM, Rosenfield CL, Knipple DC. Ethanol tolerance in the yeast Saccharomyces cerevisiae is dependent on cellular oleic acid content. Appl Environ Microbiol. 2003;69:1499-503.

29. Dinh TN, Nagahisa K, Hirasawa T, Furusawa C, Shimizu H. Adaptation of Saccharomyces cerevisiae cells to high ethanol concentration and changes in fatty acid composition of membrane and cell size. PLoS One. 2008;3:e2623. 